\title{
ChemComm
}

\section{A polyamine-modified near-infrared fluorescent probe for selective staining of live cancer cells $\dagger$}

Cite this: Chem. Commun., 2015, 51,7360

Received 24th February 2015, Accepted 9th March 2015

DOI: $10.1039 / c 5 c c 01637 a$

www.rsc.org/chemcomm

We report the synthesis of novel polyamine-modified near-infrared (NIR) probes, which show excellent water-solubility and good optical properties. One probe was taken up efficiently by living cancer cell lines whereas no staining of the non-cancer cells was observed.

Fluorophores that emit in the NIR wavelength range $(700-900 \mathrm{~nm})$ increasingly attract interest for cell and tissue imaging, ${ }^{1-8}$ as they display reduced background fluorescence and enable deep tissue penetration. In this context, indotricarbocyanines are among the most commonly used fluorophores and enclose the only clinically approved NIR dye indocyanine green, applied as a perfusion marker in, for instance, image guided oncologic surgery and lymph node imaging. ${ }^{9}$

Recently, the development of new NIR fluorescent probes that allow for tissue-selective staining due to their intrinsic chemical structures - instead of using covalently attached targeting moieties such as peptides or antibodies - has emerged as a powerful new approach. ${ }^{10}$ Attachment of cationic amino groups substantially increases the water-solubility of polyaromatic fluorophores for application as biomarkers. Several examples have been reported, including spermine-functionalized perylene bisimides, ${ }^{11,12}$ perylene bisimides with guanidinium-dendrons, ${ }^{13}$ tetraazaperopyrenes containing heterocycles with quaternized nitrogen atoms, ${ }^{14}$ multi-cationic BODIPY dyes, ${ }^{15}$ axially di-substituted silicon phthalocyanines, ${ }^{16}$ and cyanine dyes. ${ }^{17}$ Polyamine-modified probes in their protonated form display high affinity to polyanionic biopolymers such as nucleic acids and glycosaminoglycans - and are used for the detection of these molecules. ${ }^{12,18}$ In a medical context, an attractive application of polyamine-modified probes is the targeting of cancer cells, ${ }^{19}$ as most cancer cells express an overactive polyamine transport system (PTS) and tend to accumulate derivatives of the natural polyamines spermine and spermidine. ${ }^{20,21}$ F14512, a spermine-conjugate of the topoisomerase inhibitor

Universität Heidelberg, Anorganisch-Chemisches Institut, Im Neuenheimer Feld 270, 69120 Heidelberg, Germany. E-mail: Roland.Kraemer@urz.uni-heidelberg.de $\dagger$ Electronic supplementary information (ESI) available: Additional experimental data, general experimental methods, compound characterizations. See DOI: 10.1039/c5cc01637a epipodophyllotoxin, has recently completed a phase I clinical trial and is considered as a promising drug candidate for the treatment of acute myeloid leukemia. ${ }^{22}$ The structural requirements for effective cellular uptake of polyamine probes, however, remain obscure; only few probes are effectively internalized, either independently from the PTS or in strong correlation with the activity of the latter. ${ }^{23}$

Herein, we describe a new polyamine-modified indotricarbocyanine fluorescent probe with absorption and emission maxima in the near-infrared region. This probe is suitable for fluorescence imaging of living cells due to its high extinction coefficient, sufficient quantum yield, excellent water-solubility, and low cytotoxicity under the conditions applied for staining. Additionally, we show that the probe specifically targets living cancer cell lines whereas no accumulation of the dye is observed in noncancer cells.

The double carboxylic acid modified indotricarbocyanine dye backbone ${ }^{24,25} 1$ allows for highly modular chemical conjugation and was thus selected as synthetic platform. In order to evaluate the influence of different chain lengths on cellular uptake, we prepared a series of dye conjugates containing a variety of natural polyamines.

$N$-Boc-putrescine, $N^{1}, N^{5}$-di-boc-spermidine and $N^{1}, N^{4}, N^{9}$-triboc-spermine were coupled to backbone 1 via amide-bond formation. Subsequent removal of the protecting groups yielded conjugates 2-4 (Fig. 1a; see ESI $\dagger$ for synthetic details).

According to previous reports by Winkler et al., ${ }^{26}$ coupling of spermine leads to the functional analogue of a spermidine conjugate due to impairment of one $\mathrm{NH}_{2}$ group. Therefore, we additionally prepared conjugate 5 through attachment of the chemically extended spermine derivative $N^{1}, N^{4}, N^{9}, N^{13}$-tetraboc-1,16-diamino-4,9,13-triazahexadecane. The resulting dyepolyamine conjugates $\mathbf{2} \mathbf{- 5}$ showed very high extinction coefficients (260 000-285000 $\mathrm{M}^{-1} \mathrm{~cm}^{-1}$ ) and sufficient fluorescence quantum yields $(0.10-0.15)$ in combination with good solubility in aqueous solutions.

As a first step to characterize the labeling efficiency of the dyes $\mathbf{2 - 5}$ by flow cytometry, we used a cervical carcinoma cell 
a)
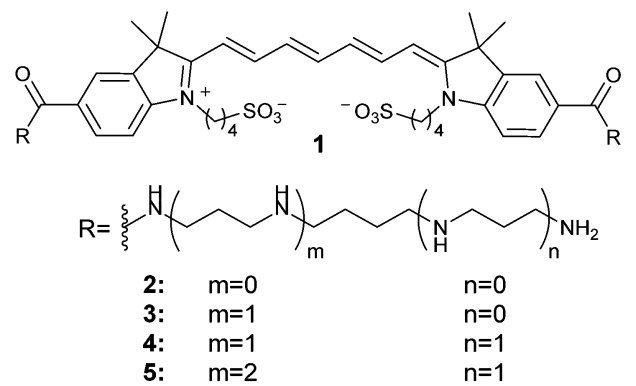

$R=\xi N(\mathrm{~N}_{\mathrm{H}}^{\mathrm{N}} \overbrace{\mathrm{m}} \mathrm{NH}_{2}$

6: $m=3$

b)

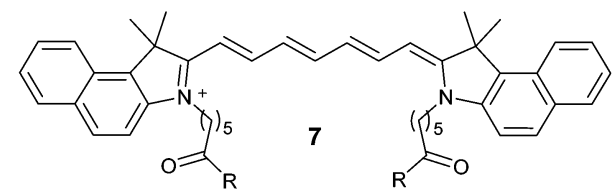<smiles>[R]C(C)(C)NC(C)(C)NCCCNC(C)(C)CCC(C)(C)NCCCN</smiles>

8: $m=2$

$\mathrm{n}=1$

Fig. 1 Structures of the herein investigated polyamine-dye conjugates based on (a) chromophore 1 and (b) chromophore 7. Counter ions are omitted.

line (HeLa) and treated the cells with $10 \mu \mathrm{M}$ solutions of the dyes for various amounts of time. The results revealed that conjugate 5 shows the highest staining efficiency while almost no staining was observed for 2 (Fig. S1, ESI $\dagger$ ). Therefore, we selected conjugate $\mathbf{5}$ for further investigations.

For a comprehensive comparison with 5, the related indotricarbocyanine derivatives 6 and $\mathbf{8}$ (Fig. 1) were also prepared. In order to study the influence of the $\mathrm{N}$-connecting alkyl moieties, we prepared 6 which contains a non-natural linear polyamine with the same number of amino groups as in $\mathbf{5}$ but with shorter $-\left(\mathrm{CH}_{2}\right)_{n}$ - fragments. Due to the promising results with the "extended" spermine conjugate 5, we also prepared conjugate 8 (Fig. 1b) which contains the same polyamine but the structurally different indotricarbocyanine core 7 ("cypate") ${ }^{27}$ that was chosen due to its analogy to the clinically applied compound indocyanine green (see ESI $\dagger$ for synthetic procedures).

The photophysical properties of 5, 6 and 8 are shown in Table 1 and Fig. 2. Dyes 5 and $\mathbf{6}$ show very high molar extinction coefficients and sufficient quantum yields which compare well to the values of related indotricarbocyanines. ${ }^{28}$ In line with the data for indocyanine green, ${ }^{29}$ the molar absorbance and quantum yield of $\mathbf{8}$ are significantly lower (Table 1).

In order to investigate the applicability of the polyaminemodified fluorophores in biological samples, we determined the cytotoxicity of the dyes in cells. Therefore, we treated HeLa cells with various concentrations $(0 \mathrm{nM}$ to $10 \mu \mathrm{M})$ of 5 and 8 for $24 \mathrm{~h}$ at $37{ }^{\circ} \mathrm{C}$ and measured the intracellular ATP levels. ${ }^{31}$ This yielded $\mathrm{IC}_{50}$ values at $5 \mu \mathrm{M}$, demonstrating the weak cellular response of the dyes (Fig. S2, ESI $\dagger$ ).

Next, to examine the labeling efficiency of the dyes in living cells, we used various cancer (HeLa and H1650) and non-cancer
Table 1 Photophysical properties of compounds 5, 6 and $\mathbf{8}$, determined in PBS (pH 7.4) with 0.1-0.5 $\mu \mathrm{M}$ dye concentrations at $20^{\circ} \mathrm{C}$

\begin{tabular}{lllll}
\hline & $\lambda_{\max }(\mathrm{abs})[\mathrm{nm}]$ & $\lambda_{\max }(\mathrm{em})[\mathrm{nm}]$ & $\varepsilon\left[\mathrm{M}^{-1} \mathrm{~cm}^{-1}\right]$ & $\varphi^{a}$ \\
\hline $\mathbf{5}$ & 755 & 781 & 268000 & 0.10 \\
$\mathbf{6}$ & 755 & 781 & 268000 & 0.11 \\
$\mathbf{8}$ & 781 & 805 & 115000 & 0.02
\end{tabular}

${ }^{a}$ Fluorescence quantum yields were determined using ICG $(\varphi=0.13$ in DMSO) as standard. ${ }^{28,30}$
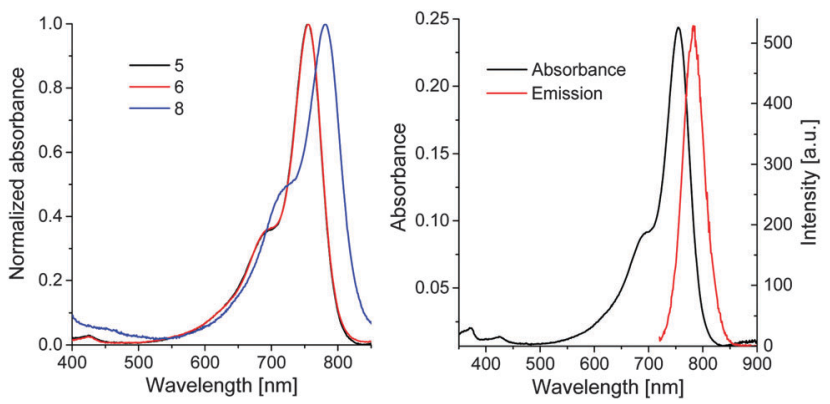

Fig. 2 Left: normalized absorption spectra of 5, 6 and 8 in PBS (pH 7.4). Right: absorption and emission spectra of $\mathbf{5}$ in PBS (pH 7.4).

(hTERT RPE-1 and HDFa) cell lines. Following Hoechst 33342 staining, we incubated the above-mentioned living cells with $10 \mu \mathrm{M}$ of dye 5 for $5 \mathrm{~min}$ at $37^{\circ} \mathrm{C}$ and imaged them by fluorescence microscopy. We observed strong staining of the cancer cell lines (Fig. 3). In contrast, in the non-cancer cells almost no accumulation of the dye was detected (Fig. 3), suggesting the function of dye $\mathbf{5}$ as a cancer-targeting fluorophore. In addition, as anticipated, derivative 6 , which contains the non-natural
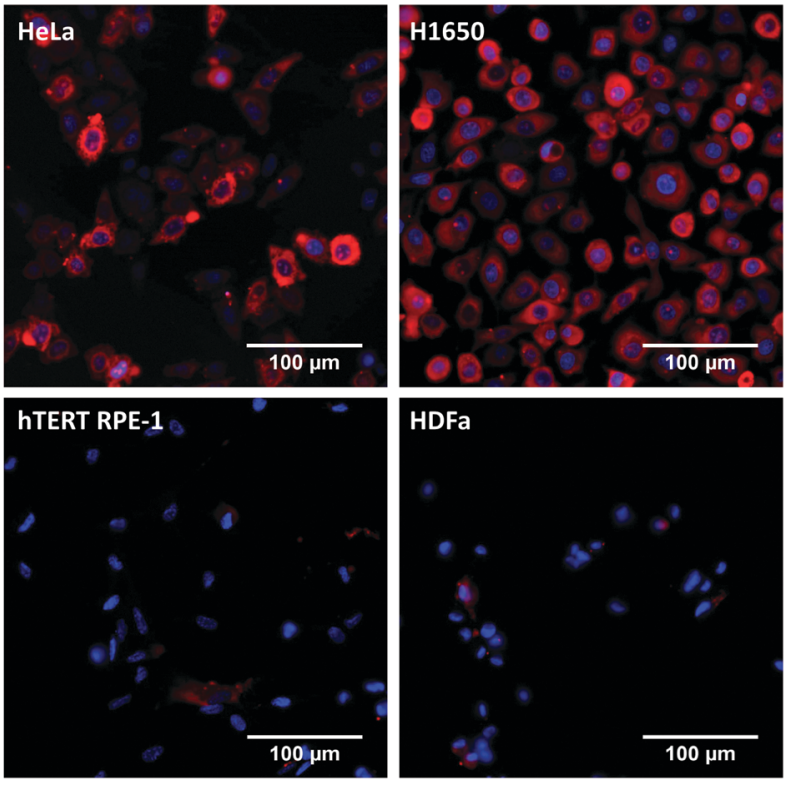

Fig. 3 Representative fluorescence microscopy images of live cancer HeLa (top, left), H1650 (top, right) and non-cancer hTERT RPE-1 (bottom, left) and HDFa (bottom, right) cells after incubation with $10 \mu \mathrm{M}$ of dye 5 for 5 min. Emission of $\mathbf{5}$ is shown in red; nuclei were co-stained with Hoechst 33342 (blue). 

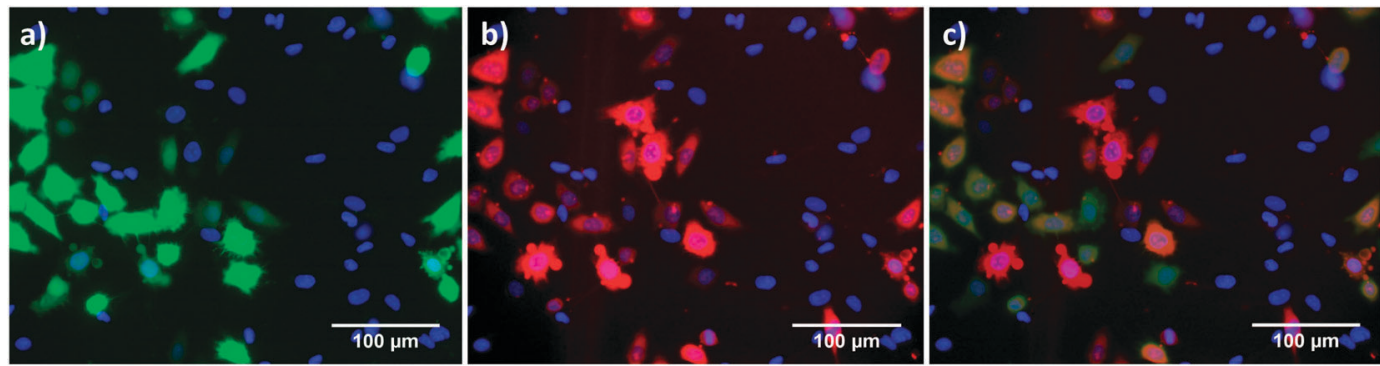

Fig. 4 Mixture of HeLa-GFP and HDFa cells. (a) Emission of GFP from HeLa cells shown in green. (b) Emission of $\mathbf{5}$ shown in red. (c) Overlay of the GFP channel and the NIR channel. Nuclei were co-stained with Hoechst 33342 (blue).

linear polyamine, did not stain any of the tested cell lines (Fig. S3, ESI $\dagger$ ).

In order to compare the staining of the cancer and noncancer cell lines simultaneously, we mixed stably GFP-expressing HeLa cells with HDFa cells in a 1:1 ratio. Following Hoechst 33342 staining, we incubated the cell mixture with $10 \mu \mathrm{M}$ of 5 for $5 \mathrm{~min}$ at $37{ }^{\circ} \mathrm{C}$ and imaged by fluorescence microscopy. We observed an almost 100\% co-localization (Fig. 4c) of GFP signals coming from HeLa cells (green, Fig. 4a) with dye 5 (red, Fig. 4b). On the other hand, HDFa cells, which can be seen with their nuclei staining (blue), did not accumulate 5 (Fig. 4b), as previously observed (Fig. 3).

In view of potential applications of the dyes in vivo or in blood samples, we incubated HeLa and hTERT RPE-1 cells with solutions of 5 and 8 in pooled human plasma. For $\mathbf{5}$, we observed a more than five times higher staining efficiency for the cancer cells compared to the non-cancer cells $(p=0.009)$ (Fig. 5a). In contrast, 8 did not show a pronounced selectivity for one cell line over the other. Similar results were also observed for $\mathbf{8}$ by fluorescence microscopy (Fig. S4, ESI $\dagger$ ).

Next, we aimed to clarify the role of an overexpressed PTS in the selective uptake of $\mathbf{5}$ in cancer cells. Following the inhibition of the PTS in HeLa with benzylviologen ${ }^{32}$ for 24 hours, we incubated the cells with $\mathbf{5}$ and measured the fluorescence intensity by flow cytometry. Inhibition of the PTS resulted in a $62 \%$ of reduction of uptake of 5 , compared to untreated cells a)

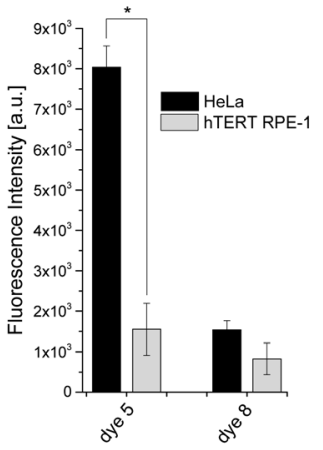

b)

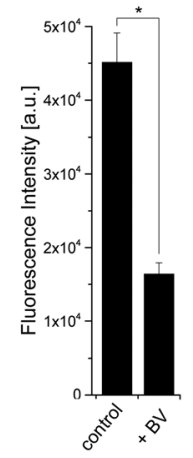

Fig. 5 Mean fluorescence intensities determined by flow cytometry of (a) living HeLa and hTERT RPE-1 cells after incubation with $10 \mu \mathrm{M}$ of dyes 5 and $\mathbf{8}$ in pooled human plasma for 5 min, (b) living HeLa cells after treatment with $10 \mu \mathrm{M}$ of dye $\mathbf{5}$ in FCS-free RPMI with (+BV) or without (control) the PTS-inhibitor benzylviologen. * = statistically significant.
( $p=0.002$ ) (Fig. 5b), confirming the major role of PTS in the uptake of this particular polyamine dye.

In this work, a series of polyamine-modified NIR-fluorescent probes that exhibit advantageous optical properties and good water-solubility were developed. The most interesting candidates contain an "extended" spermine moiety and were subject of further investigations within living cells. While all of the investigated derivatives proved to have low cytotoxicity under the abovementioned treatment conditions, only 5 showed enhanced staining of live cancer cell lines whereas non-cancer cells remained unstained. Additionally, we were able to demonstrate the selective staining of cancer cells in human plasma. In order to broaden the applicability of our system, future experiments will focus on systematic investigations with a variety of cell lines.

With regard to its chemical and optical properties, we consider the development of $\mathbf{5}$ as an important step towards a simple smallmolecule probe that combines the polyamine-vector triggered, selective uptake by live cancer cells with minimized interference with cellular autofluorescence due to NIR fluorescence. Such probes may facilitate the fluorescence scanning microscopic identification of individual tumor cells in the presence of a large excess of other cells, as required for the quantification of circulating tumor cells in blood samples ${ }^{33}$ for early diagnosis.

SGK is grateful to the Stiftung der deutschen Wirtschaft (sdw) for a PhD fellowship.

The authors thank Dr Tautvydas Lisauskas, Dr Holger Erfle, Dominik Brox and Prof. Dirk-Peter Herten for helping with fluorescence microscopy, Elisabeth Treutlein and Ana Lucía Meza Chincha for synthetic support and Berati Cerikan for the hTERT RPE-1 and HeLa T-REX stable LAPtag cell lines.

\section{Notes and references}

1 Z. Guo, S. Park, J. Yoon and I. Shin, Chem. Soc. Rev., 2014, 43, 16-29.

2 K. Umezawa, D. Citterio and K. Suzuki, Anal. Sci., 2014, 30, 327-349.

3 L. Yuan, W. Lin, K. Zheng, L. He and W. Huang, Chem. Soc. Rev., 2013, 42, 622-661.

4 G. Lukinavičius, K. Umezawa, N. Olivier, A. Honigmann, G. Yang, T. Plass, V. Mueller, L. Reymond, I. R. Corrêa Jr, Z.-G. Luo, C. Schultz, E. A. Lemke, P. Heppenstall, C. Eggeling, S. Manley and K. Johnsson, Nat. Chem., 2013, 5, 132-139.

5 J. V. Frangioni, Curr. Opin. Chem. Biol., 2003, 7, 626-634.

6 M. Bai and D. J. Bornhop, Curr. Med. Chem., 2012, 19, 4742-4785.

7 S. Luo, E. Zhang, Y. Su, T. Cheng and C. Shi, Biomaterials, 2011, 32, 7127-7138.

8 X. Tan, S. Luo, D. Wang, Y. Su, T. Cheng and C. Shi, Biomaterials, 2012, 33, 2230-2239. 
9 E. M. Sevick-Muraca, Annu. Rev. Med., 2012, 63, 217-231.

10 H. Hyun, M. H. Park, E. A. Owens, H. Wada, M. Henary, H. J. M. Handgraaf, A. L. Vahrmeijer, J. V. Frangioni and H. S. Choi, Nat. Med., 2015, 21, 192-197.

11 S. Rehm, V. Stepanenko, X. Zhang, T. H. Rehm and F. Würthner, Chem. - Eur. J., 2010, 16, 3372-3382.

12 H. Szelke, S. Schübel, J. Harenberg and R. Krämer, Chem. Commun., 2010, 46, 1667-1669.

13 J. Zhou, J. Zhang, Y. Lai, Z. Zhou, Y. Zhao, H. Wang and Z. Wang, New J. Chem., 2013, 37, 2983-2986.

14 L. Hahn, S. Öz, H. Wadepohl and L. H. Gade, Chem. Commun., 2014, 50, 4941-4943.

15 J. Bartelmess and W. W. Weare, Dyes Pigm., 2013, 97, 1-8.

16 Z. Biyıklığlu, Dyes Pigm., 2013, 99, 59-66.

17 Y. Guminski, T. Imbert, S. Pesnel, A. Pillon, A. Le Pape and S. Lerondel, WO 2012/069607 A1, 2012.

18 See for example H. Szelke, S. Schübel, J. Harenberg and R. Krämer, Bioorg. Med. Chem. Lett., 2010, 20, 1445-1447.

19 P. M. Cullis, R. E. Green, L. Merson-Davies and N. Travis, Chem. Biol., 1999, 6, 717-729.

20 A. G. Palmer and H. M. Wallace, Amino Acids, 2010, 38, 415-422.

21 N. Seiler, J. G. Delcros and J. P. Moulinoux, Int. J. Biochem. Cell Biol., 1996, 28, 843-861.

22 A. Kruczinski, A. Pillon, L. Creancier, I. Vandenberghe, B. Gomes, V. Brel, E. Fournier, J.-P. Annereau, E. Currie, Y. Guminski, D. Bonnet, C. Bailly and N. Guilbaud, Leukemia, 2013, 27, 2139-2148.
23 For a systematic study see Y. Guminski, M. Grousseaud, S. Cugnasse, V. Brel, J.-P. Annereau, S. Vispe, N. Guilbaud, J.-M. Barret, C. Bailly and T. Imbert, Bioorg. Med. Chem. Lett., 2009, 19, 2474-2477.

24 E. Terpetschnig, H. Szmacinski, A. Ozinskas and J. Lakowicz, Anal. Biochem., 1994, 217, 197-204.

25 C. Pavlik, N. Biswal, F. Gaenzler, M. Morton, L. Kuhn, K. Claffey, Q. Zhu and M. Smith, Dyes Pigm., 2011, 89, 9-15.

26 J. Winkler, K. Saadat, M. Díaz-Gavilán, E. Urban and C. R. Noe, Eur. J. Med. Chem., 2009, 44, 670-677.

27 S. Achilefu, H. N. Jimenez, R. B. Dorshow, J. E. Bugaj, E. G. Webb, R. R. Wilhelm, R. Rajagopalan, J. Johler and J. L. Erion, J. Med. Chem., 2002, 45, 2003-2015.

28 K. Licha, B. Riefke, V. Ntziachristos, A. Becker, B. Chance and W. Semmler, Photochem. Photobiol., 2000, 72, 392-398.

29 O. G. Björnsson, R. Murphy, V. S. Chadwick and S. Björnsson, J. Clin. Chem. Clin. Biochem., 1983, 21, 453-458.

30 R. C. Benson and H. A. Kues, J. Chem. Eng. Data, 1977, 22, 379-383.

31 S. P. Crouch, R. Kozlowski, K. J. Slater and J. Flechter, J. Immunol. Methods, 1993, 160, 81-88.

32 M. Wolf, W. E. Hull, W. Mier, S. Heiland, U. Bauder-Wüst, R. Kinscherf, U. Haberkorn and M. Eisenhut, J. Med. Chem., 2007, 50, 139-148.

33 C. Shao, C.-P. Liao, P. Hu, C.-Y. Chu, L. Zhang, M. H. T. Bui, C. S. Ng, D. Y. Josephson, B. Knudsen, M. Tighiouart, H. L. Kim, L. W. K. Chung, R. Wang and E. M. Posadas, PLoS One, 2014, 9, e88967. 